\title{
One-Pot Synthesis of Tetracyanocyclopropane Derivatives Using Hexamethylenetetramine-Bromine (HMTAB)
}

\author{
Majid Momahed Heravi (D), Hossein Abdi Oskooie, Zahra Latifi , Hoda Hamidi \\ Department of Physics and Chemistry, Alzahra University, Tehran,P.0. Box 1993891176, Iran \\ *E-mail address: hamidi_hoda@yahoo.com, Corresponding author: Tel.: +982188044051; Fax: +982188041344
}

Received: 25 September 2018, Revised: 17 October 2018, Accepted: 8 November 2018

\begin{abstract}
A B S T R A C T
Herein a direct method for synthesis of tetracyanocyclopropanes by the treatment of carbonyl compounds, malononitrile and hexamethylenetetramine-bromine (HMTAB) is described in the presence of catalytic amount of DABCO in ethanol media at room temperature.
\end{abstract}

Keywords: Tetracyanocyclopropane derivatives, hexamethylenetetramine-bromine (HMTAB), DABCO.

GR A P H I C A L ABSTRACT

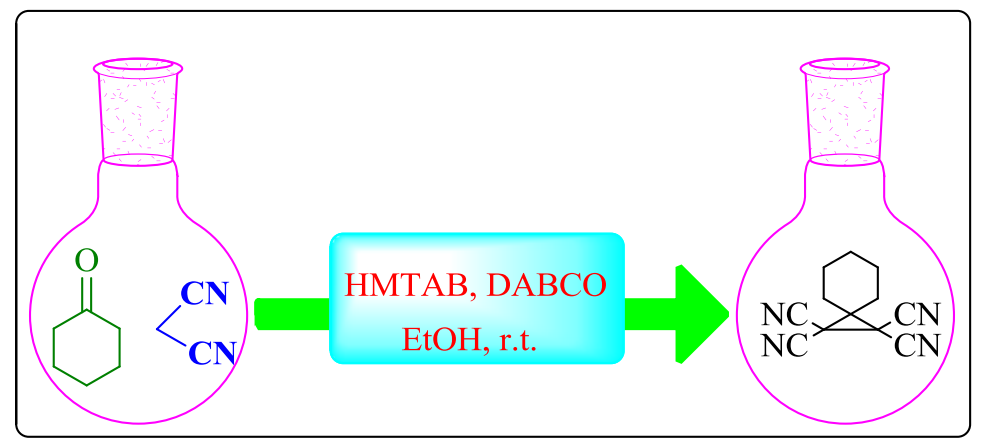

\section{Introduction}

Developing of new methodologies of particularly advantages in the last few decades, has been growing chemical synthesis horizons of organic compounds. Cyclopropane derivatives have been attract many interestings due to various biological applications such as antitumour, antimicrobial, antiviral, antioxidant, antifungal, antitubercular, herbicidal activities and enzymes inhibitory [1-8].

The compounds illustrated in Figure 1 as potential antitumor and anticancer agents. Cyclopropane derivatives I, III shown active antitumor agent and Polo-Like Kinase 4 Inhibitors respectively, also II has significant activity against human prostate cancer cells [9-
12]. Reactions occurred in the cyclopropane synthesis include two major intramolecular and intermolecular (addition of carbenes to olefins or Michael initiated ring closure (MIRC)) cyclization groups.

Application of HMTAB containing bromine source instead of other bromination agents such as NBS (N-bromosuccinimide) [13], $\mathrm{Br}_{2} / \mathrm{SO}_{2} \mathrm{Cl}_{2}$ [14], N,N,N,N-tetrabromobenzene-1,3disulfonylamide [15] and alkyl halides (e.g. $\mathrm{NaBr}$ ), is also seen to some extent but is not common between organic chemists. Indeed, we wish to disclose a capable compound for bromination of knovenogel intermediate in synthesis of cyclopropanes. 

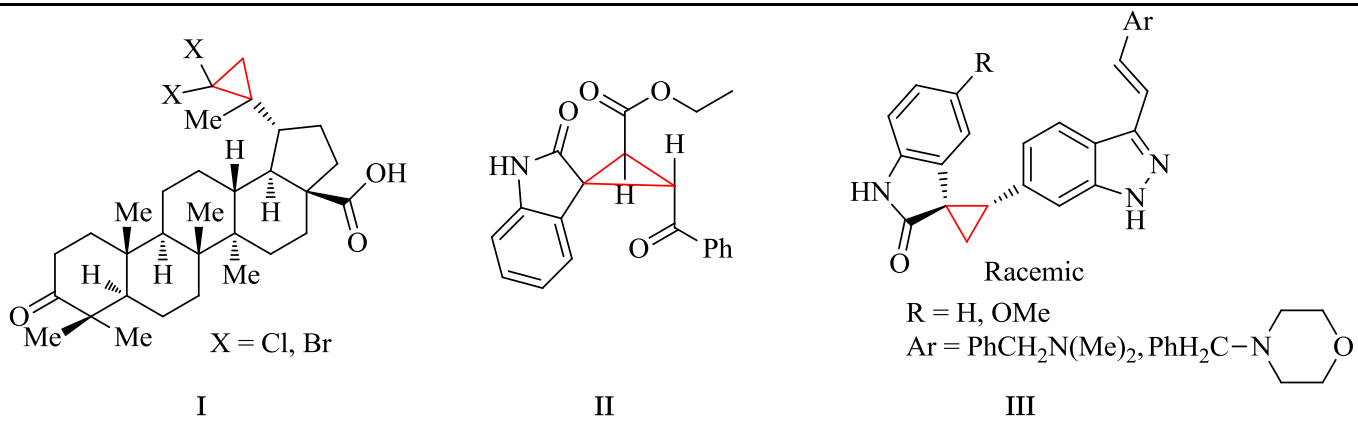

Figure 1. Some biologically active cyclopropane compounds as antitumor (I, III) and anticancer (II).

The earliest method to cyclopropanation of olefins revisited to 1958, only when Simmons and Smith was discovered the treatment of $\mathrm{CH}_{2} \mathrm{I}_{2}$ with $\mathrm{Zn}(\mathrm{Cu})$ gave rise to the best reagent for this purpose [16]. Among different methods have been reported so far, Elinson et al was carried out such reactions using bromine in the presence of 1.2 equiv of EtONa in ethanol media [17]. Also, a galvanostatic electrolysis technique has been modified due to the synthesis of 1chloro-2-cyanocyclopropanecarboxylic acid stereoisomers by Neverov et al [18].

In the light of these studies about tetracyanocyclopropanes, we suggested an efficient novel one pot synthesis of tetracyanocyclopropanes by the reaction of carbonyl compounds, malononitrile and hexamethylenetetramine-bromine (HMTAB) as useful reagent in the presence of basic catalyst. Among many examined catalyst, 1,4diazabicyclo[2.2.2] octane (DABCO) is selected as an available and operative catalyst in this protocol.

\section{Result and disscution}

The reaction of carbonyl compounds, malononitrile and prepared hexamethylenetetramine-bromine

(HMTAB) according the procedure [19] as a model reaction in order to synthesis tetracyanocyclopropanes is demonstrated in Scheme 1. The assessment of various catalysts such as $\mathrm{NaOH}, \mathrm{KOH}$, morpholine, and DABCO is studied. It is noteworthy to mention that the reaction between ketone or aldehyde and malononitrile confirmed in high yields using DABCO as catalyst. The results showed that 0.13 mmol is the optimal amount of catalyst and use of higher amount did not increase the yield (Table 1). Various carbonyl compounds were reacted with malononitrile to prove the generality of the method in the presence of HMTAB as an efficient reagent to achieve the products in high yields (Table 2).

Table 1. Optimization of amount of catalyst for compound 3a

\begin{tabular}{cccc}
\hline Entry & Catalyst & Time (h) & Yield (\%) \\
\hline 1 & DABCO(0.8 mm0l) & 2.5 & 83 \\
2 & DABCO(1.0 mm0l) & 2.5 & 85 \\
3 & DABCO $(1.2 \mathrm{~mm} 0 \mathrm{l})$ & 2 & 90 \\
4 & DABCO $(1.3 \mathrm{~mm} 0 \mathrm{l})$ & 2 & 90 \\
\hline
\end{tabular}

The following scheme (Scheme 2) is suggested for the alteration of benzylidene- or alkylidenemalononitriles 1a-i and malononitrile 2 to tetracyanocyclopropane derivatives $\mathbf{3 a - i}$. The knovenogel reaction of malononitrile with carbonyl compounds give benzylidene- or alkylidenemalononitrile $\mathbf{1}$ which undergoes of 


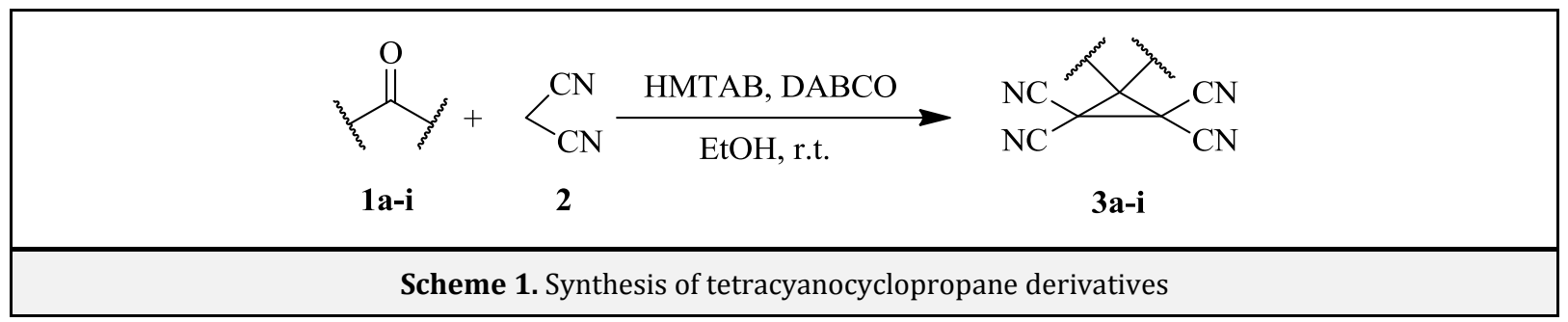

Table 2. Cyanocyclopropanation of carbonyl compounds

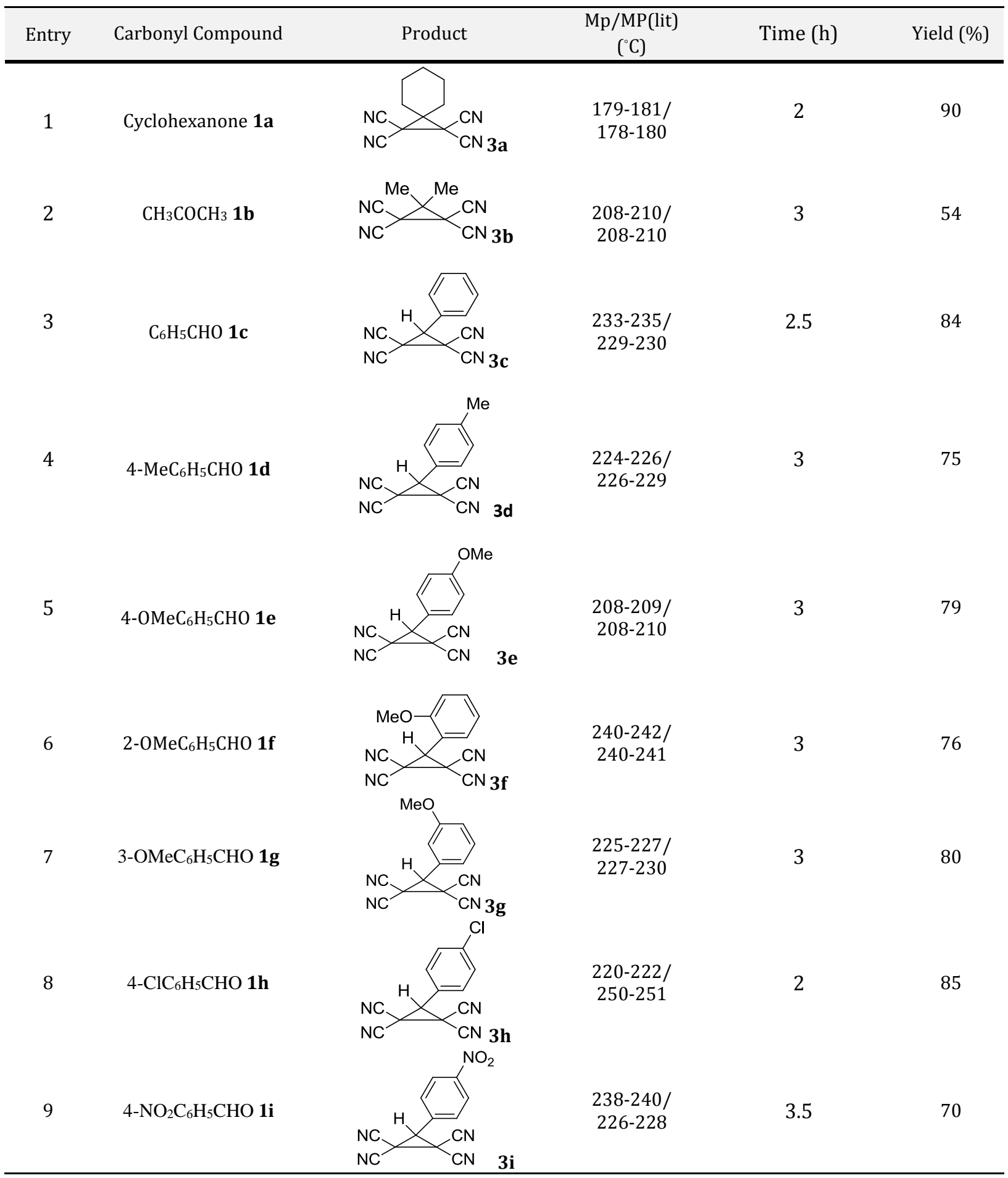


Michael addition with second mole of malononitrile in the aide of catalytic effect of DABCO base. The bromination of anion 4 with the action of HMTAB as a halogen source results intermediate $\mathbf{5}$. The last step of the mechanism is formation of tetracyanocyclopropane $\mathbf{3}$ by subsequent cyclisation of $\mathbf{5}$ in ethanol solution.

\section{Experimental}

\subsection{Chemicals and apparatus}

All chemicals and solvents were utilized without further purification. Melting points were measured by Bamstead Electrothermal 9200 apparatus. 1HNMR: Bruker DRX-500-Avance instrument; IR Spectra: FT-IR Bruker Tensor 27; EI-MS: Agilent-Technologies-(HP)-5937 mass spectrometer.

\subsection{General Procedure}

DABCO (1.2 mmol) which solved in 2cc ethanol was added to a $5 \mathrm{cc}$ ethanolic mixture of cyclohexanone 1 ( $1 \mathrm{mmol}$ ) and malononitrile 2 ( $1 \mathrm{mmol}$ ) in $25 \mathrm{cc}$ bottom round flask. Then, after stirring for 5 minutes at room temperature, 2 mmol of HMTAB was added during 3 minutes. After completion of the reaction which monitored by TLC, the crude product was filtered and washed with warm water and finally the resulting white powder was dried and recrystallized with ethanol.

Compound $3 \boldsymbol{a}$ (1,1,2,2-Tetracyanospiro [2.5] octane): White powder, m.p. $179-181{ }^{\circ} \mathrm{C}$, IR (KBr): 2957, 2934, 2902, 2859, 2261, 2253, 1450, 1375, 1276, 1129 cm-1, 1H NMR (400 MHz, DMSO): 1.51-1.57 (m, 2H, $\mathrm{CH}_{2}$ ), 1.65-1.79 (m, 4H, CH2), 1.85-1.98 (m, 4H, $\left.\mathrm{CH}_{2}\right)$.

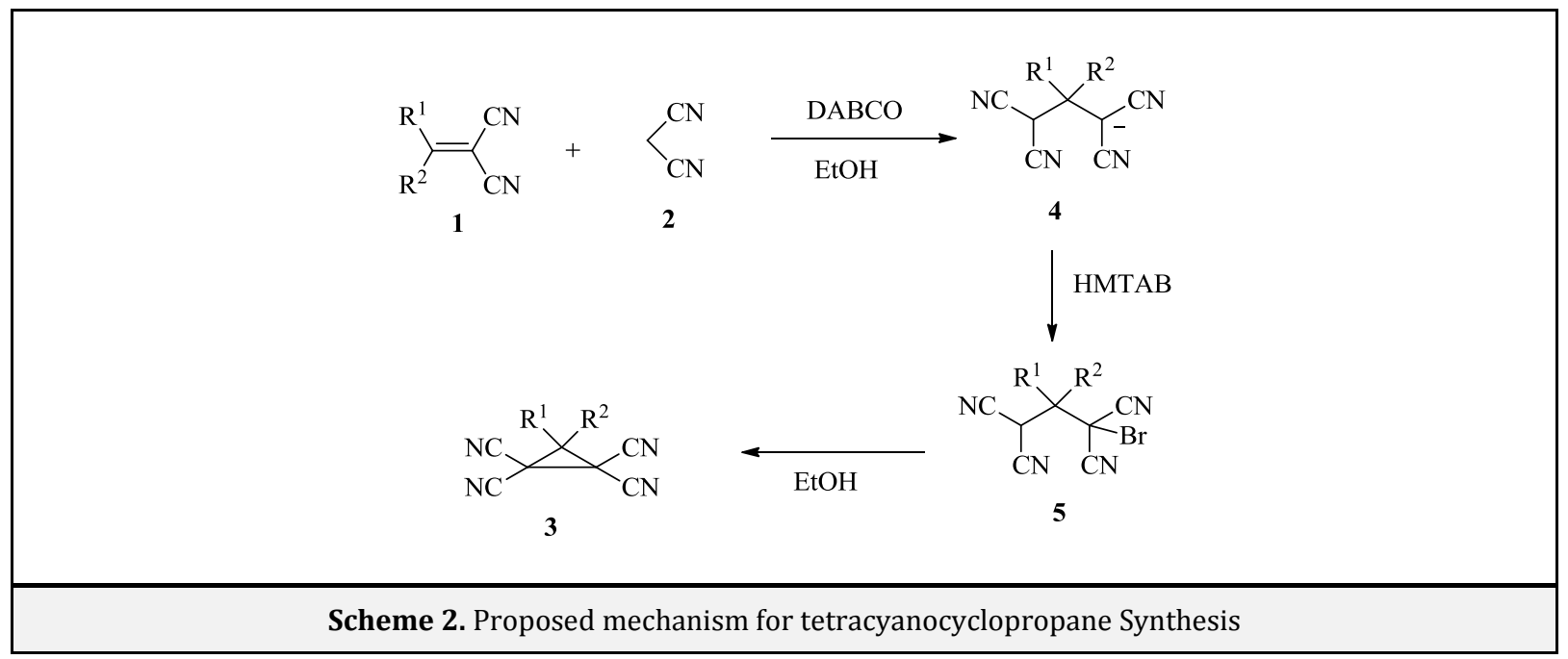

\section{Conclusion}

In conclusion, we described a one pot transformation of carbonylcompounds, malononitril and $\mathrm{HMTAB}$ as a useful reagent to tetracyanocyclopropane derivatives in high yield by reducing time of the reaction. Among many examined catalyst, 1,4diazabicyclo[2.2.2] octane (DABCO) is selected as an available and operative catalyst in this protocol.
Acknowledgment The authors are grateful for the partial financial support from Alzahra University research council via special grant.

\section{ORCID}

Majid M. Heravi (D): 0000-0003-2978-1157

Hoda Hamidi iD: 0000-0002-5019-8591

\section{References}

[1]. Y. Baba, G. Saha, S. Nakao, C. Iwata, T. Tanaka, T. Ibuka, H. Ohishi, Y. Takemoto, J. Org. Chem., 2001, 66, 81-88.

[2]. D.L. Boger, T.V. Hughes, M.P. Hedrick, J. Org. Chem., 2001, 66, 2207-2216. 
[3]. D.W. Graham, W.T. Ashton, L. Barash, J.E. Brown, R.D. Brown, L.F. Canning, A. Chen, J.P. Springer, E.F. Rogers, J. Med. Chem., 1987, 30, 1074-1090.

[4]. S. Yoshida, T.C. Rosen, O.G.J. Meyer, M.J. Sloan, S. Ye, G. Haufe, K.L. Kirk, Bioorg. Med. Chem., 2004, 12, 2645-2652.

[5]. M.P. Toraskar, V.J. Kadam, V.M. Kulkarni, Int. J. Pharm. Pharm. Sci., 2010, 2, 132-133.

[6]. K. Othmer, Kirk-Othmer Encyclopedia of Chemical Technology, Insecticides, New York, Wiley, 1965, p. 685.

[7]. D. Namrata, T. Neetu, V.K. Tiwari, C. Vinita, Y.K. Manju, A. Srivastava, A. Giakwad, S. Sinha, R.P. Tripathi, Bioorg. Med. Chem. Lett., 2005, 15, 4526-4530.

[8] K. Yamaguchi, Y. Kazuta, K. Hirano, S. Yamada, A. Matsuda, S. Shuto, Bioorg. Med. Chem., 2008, 16, 8875-8881.

[9] C.N. Reddy, V.L. Nayak, G.S. Mani, J.S. Kapure, P.R. Adiyala, R.A. Maurya, A. Kamal, Bioorg. Med. Chem. Lett., 2015, 25, 4580-4586.

[10] R. Laufer, B. Forrest, S.W. Li, P. Sampson, Y. Liu, L. Edwards, Y. Lang, D. Awrey, V. Mao, O. Plotnikova, G. Leung, R. Hodgson, I. Beletskaya, J. Mason, S. Wei, X. Luo, V. Nadeem, M. Feher, R. Kiarash, E. Green, T. W. Mak, J. G. Pan, H.W. Pauls, J. Med. Chem., 2013, 56, 6069-6087.
[11] P.B. Sampson, Y. Liu, B. Forrest, G. Cumming, S.W. Li, N.K. Patel, L. Edwards, R. Laufer, M. Feher, F. Ban, D.E. Awrey, G. Mao, O. Plotnikova, R. Hodgson, I. Beletskaya, J.M. Mason, X. Luo, V. Nadeem, X. Wei, R. Kiarash, B. Madeira, P. Huang, T. W. Mak, G. Pan, H.W. Pauls, J. Med. Chem., 2015, 58, 147-169.

[12] K.K. Ajay, R.K.M. Lokanatha, K.G. Vasanth, B.N. Mylarappa, Int. J. Pharm. Pharm. Sci., 2012, 4, 564-568.

[13] M. Bagheri, N. Azizi, M.R. Saidi, Can. J. Chem., 2005, 83, 146-149.

[14] J.M. Gnaim, R.A. Sheldon, Tetrahedron Lett., 2005, 46, 4465-4468.

[15] R. Ghorbani-Vaghei, H. Jalili, Synthesis, 2005, 7, 1099-1102.

[16] H.E. Simmons, R.D. Smith, J. Am. Chem. Soc., 1958, 80, 5323-5324.

[17] S.V. Neverov, V.L. Sigachevaz, V.A. Petrosyan, Russian J. Electrochem. 2011, 47, 1134-1138.

[18] M.N. Elinson, K.F. Sergey, N.O. Stepanov, N.V. Anatolii, I.N. Gennady, Tetrahedron, 2008, 64, 708-713.

[19] M.M. Heravi, N. Abdolhosseini, H.A. Oskooie, Tetrahedron Lett., 2005, 46, 8959-8963.

How to cite this manuscript: M.M. Heravi, H. Hamidi, N. Karimi, A. Amouchi, Caro's Acid-Silica Gel Catalyzed Regioselective Ring Opening of Epoxides with Indoles and Imidazoles under Solvent-Free Conditions, Adv. J. Chem. A, 2018, 1, 7-11. 